\title{
Perspective
}

\section{Role of the blood-brain barrier in rabies virus infection and protection}

\author{
Lihua Wang, Yuxi Cao, Qing Tang, Guodong Liang \\ State Key Laboratory for Infectious Disease Prevention and Control, Collaborative Innovation Center for Diagnosis and \\ Treatment of Infectious Diseases, National Institute for Viral Disease Control and Prevention, Chinese Center for Disease Control \\ and Prevention, Beijing 102206, China \\ $\triangle$ Correspondence: gdliang@hotmail.com
}

Rabies is an acute, progressive encephalitis caused by infection with rabies virus (RABV). It is one of the most important zoonotic infections and causes more than 70,000 human deaths annually (http://www. rabiescontrol.net). It has long been held that a rabies infection is lethal in humans once the causative RABV reaches the central nervous system (CNS); however, this concept was challenged by the recent recovery of a small number of rabies patients. An analysis of these patients revealed that the bloodbrain barrier (BBB) played a major role in protection against the virus. The main reason for the survival of these patients was enhanced BBB permeability after infection with the causative agent (usually batoriginated RABV showing reduced pathogenicity), which allowed immune cells to enter the tissues of the CNS and clear the infection (Willoughby et al., 2005). These findings have been confirmed in animal infection experiments (Wang et al., 2005; Roy and Hooper, 2007, 2008; Faber et al., 2009). Thus, the BBB has attracted the attention of scientists interested in the pathogenesis of, and therapeutic approaches, for rabies. This paper introduces the role of the BBB in rabies infections and protection of the CNS and provides insight into future treatments for patients with clinical rabies.

\section{THE INTEGRITY OF THE BBB IS MAINTAINED DURING LETHAL RABV INFECTIONS}

The BBB, a membranic structure composed of endothelial cells, separates circulating blood from cerebrospinal fluid (CSF), which protects the tissues of the CNS from circulating cells and various factors (Pachter et al., 2003). Magnetic resonance imaging studies have shown that the BBB remains relatively intact until the end stages of rabies in humans. An intact BBB prevents immune effectors from entering the CNS to clear the virus, and leads to disease in lethal RABV infections (Laothamatas et al., 2003). Experiments in mice demonstrated a lack of immune effectors, including $\mathrm{T}$ and $\mathrm{B}$ cells, in the tissues of the CNS following intradermal inoculation with lethal RABVs (Phares et al., 2006, 2007;
Roy and Hooper, 2007). A subsequent study showed that a lethal infection with pathogenic RABV could be prevented by increasing the permeability of the BBB in infected animals to enable the entrance of immune effectors into CNS tissue and promote virus clearance (Roy and Hooper, 2008). This may explain why virus-neutralizing antibodies (VNA), which are important for clearing viruses from the CNS, are generally not detected in the CNS of rabies patients ( $\mathrm{He}$ machudha et al., 2013). These findings were supported by the absence of neurovascular permeability in mice infected with lethal RABVs (Roy and Hooper, 2007), and led us to conclude that lethal RABVs evade immune clearance from CNS tissues through the maintenance of BBB integrity (Roy and Hooper, 2007; Fabis et al., 2008).

\section{BBB PERMEABILITY IS ENHANCED DURING AN ATTENUATED RABV INFECTION}

The survival of a nonvaccinated young girl with encephalitic rabies was described in 2004 (Jackson et al., 2005; Willoughby et al., 2005). Since then, additional similar cases have been reported, most involving patients who were infected with bat RABV variants (US CDC, 2012; Hemachudha et al., 2013). Laboratory studies showed that the main reason for the survival of these patients was the presence of VNA in the CNS. Infection with bat-originated RABV (which has lower pathogenicity than dog-originated RABV) in these cases enhanced BBB permeability, allowing immune cell entry into CNS tissues and clearance of the infection from the CNS (Willoughby et al., 2005; Jackson 
et al., 2009). This finding has kindled hope for new rabies treatments, and several studies have been performed to determine the role of the BBB in rabies infection and protection. In animal experiments, Phares et al. (2006, 2007) reported that the BBB was opened following infection with the lab-attenuated virus CVS-F3, and transiently increased BBB permeability has been found to be helpful in the clearance of attenuated RABV from the CNS; however, the lethal silver-haired bat RABV cannot induce changes in BBB permeability. Roy and Hooper (2008) found that immune effectors, including $\mathrm{T}$ and $\mathrm{B}$ cells, appeared in the CNS tissues of mice following the spread of attenuated RABV to the CNS, but not in the CNS of animals infected with wild-type virus. Moreover, the fluidphase permeability associated with immune cell infiltration into the CNS tissues of mice infected with attenuated RABV was undetectable in mice infected with wild-type RABV (Roy and Hooper, 2008). During the course of the clearance of attenuated RABV from CNS tissues, the BBB becomes permeable to fluid-phase markers (Fabis et al., 2008). These findings indicate that BBB opening is associated with the clearance of attenuated RABV from the CNS, and that a failure to open the BBB leads to disease in cases of lethal RABV infection (Phares et al., 2006, 2007; Roy and Hooper, 2007). This is consistent with the detection of VNA in the CSF of some bat-originated RABV-associated rabies patients (Willoughby et al., 2005; Hemachudha et al., 2013).

\section{THE MECHANISM OF BBB PERMEABILITY ALTERATION AFTER INFECTION WITH ATTENUATED RABV}

The mechanism leading to changes in BBB permeability after infection with attenuated RABV is not entirely clear. It was hypothesized that TNF- $\alpha$ contributes to alterations in BBB integrity since TNF- $\alpha$ can enhance the expression of ICAM-1 (an important adhesion molecule) on endothelial cells (Phares et al., 2007). However, the BBB permeability and virus replication are similar in wildtype and TNF- $\alpha$ knockout mice, indicating that TNF- $\alpha$ expression is not directly required for opening of the BBB (Phares et al., 2007).

The role of chemokines in the enhancement of BBB permeability and infiltration of inflammatory cells into the CNS after attenuated RABV infection has attracted significant attention in recent years. Chemokines are a family of structurally related proteins that are expressed by almost all types of nucleated cells and that mediate leukocyte activation and/or chemotactic activities (Zlotnik et al., 2000). It has been found that attenuated RABV is capable of inducing chemokine expression as part of innate immune responses, which benefit the host by initiating the infiltration of inflammatory cells into the CNS, enhancing BBB permeability, and clearing the virus from the CNS. However, lethal RABV fails to induce the expression of chemokines and other innate immune molecules (Sarmento et al., 2005; Wang et al., 2005; Kuang et al., 2009). Indeed, overexpression of chemokines with the ability to activate dendritic cells increased RABV immunogenicity and provided better protection (Zhao et al., 2010; Wen et al., 2011). Among these chemokines, the expression of IP-10 was best correlated with the infiltration of inflammatory cells into the CNS and enhancement of BBB permeability. IP-10 recruits inflammatory cells (mainly $T$ and natural killer cells) across the BBB into the CNS, and this function is intimately linked to BBB integrity. The treatment of mice with anti-IP-10 antibodies prevented the recruitment of inflammatory cells to the CNS and resulted in a significant decrease in the numbers of $\mathrm{CD}^{+}$ and $\mathrm{CD}^{+} \mathrm{T}$ lymphocytes infiltrating into the CNS (Liu et al., 2001). Stamatovic et al. (2003) reported that MCP-1 appears to contribute to breakdown of the BBB. MCP-1 decreased transepithelial electrical resistance and increased permeability to insulin in mBMEC monolayers from wild-type mice, but not across mBMEC monolayers from mutant mice that lacked CCR2 (a receptor for MCP-1). MCP-1 elicited rearrangement of the actin cytoskeleton, visible gaps between cells, and the redistribution of tight junction proteins (Stamatovic et al., 2003). MIP1- $\alpha$ may facilitate the entry of inflammatory cells into the CNS by altering the integrity of the BBB and contribute to the pathogenesis of autoimmune and infectious diseases. Treatment of mice with anti-MIP1- $\alpha$ antibodies prevents the recruitment of inflammatory cells into the CNS (Mucke et al., 1993; Tanabe et al., 1997). Man et al. (2007) found that the interaction of MIP1- $\alpha$ and CCR5 triggered the opening of endothelial tight junctions in the BBB. Zozulya et al. (2007) demonstrated that MIP1- $\alpha$ increases the transmigration of dendritic cells across brain microvessel endothelial monolayers.

To further explore the role of chemokines in RABV infections, the chemokine MIP1- $\alpha$, RANTES, or IP-10 was expressed in the genome of the RABV HEP-Flurry strain. HEP-MIP1-a enhanced BBB permeability temporarily, while HEP-RANTES and HEP-IP10 induced more extensive and prolonged enhancement of BBB permeability (Zhao et al., 2010). Thus, these studies indicate that transient chemokine expression may promote RABV attenuation. These data confirm that chemokines could be used to enhance the BBB and to allow RABV-specific immunity to enter the CNS to prevent the development of rabies.

\section{FUTURE PERSPECTIVES}

Rabies remains a public health problem worldwide, especially in developing countries. Around 3.9 billion people are at risk worldwide, and $>150$ countries are affected (Yin et al., 2013). China is a high-risk environment for rabies, with the number of related deaths being second only to India, and where dogs continue to serve as the main reservoir of disease transmission to humans (Cai et al., 2011; Lv et al., 2012; Zhu et al., 2012). Based on scientific and technological developments over the last few decades, insight into the pathogenesis of rabies has been gained, which will facilitate the development of a therapeutic strategy for rabies. 
Based on current knowledge, for rabies infection and protection, there are two important factors: VNA in the periphery and enhanced BBB permeability. Infection with live-attenuated RABV can induce RABV-specific VNA responses and enhance BBB permeability. Li et al. (2012) developed a live-attenuated triple-glycoprotein RABV variant (TriGAS), which can trigger the clearance of wild-type RABV from the CNS through the rapid induction of genes relevant to adaptive immunity in CNS tissues, and proposed its use in post-exposure treatment. Wu et al. (2011) found that liveattenuated RABV co-infected with street RABV protected animals against rabies. In addition, chemokines could be used to enhance the BBB and to allow RABVspecific immunity to enter the CNS to prevent the development of rabies. Thus, recombinant RABVs expressing chemokines could be used not only for pre- and post-exposure immunization but also for therapy in clinical rabies. As we continue to improve our understanding of the basic mechanisms underlying the pathogenesis of rabies in humans and animals, those with protective roles could be exploited for the development of novel RABV vaccines and therapeutic agents. Hopefully, we will conquer this ancient disease in the near future.

\section{FOOTNOTES}

This work is supported by grants from National Natural Science Foundation of China (Grant No. 81290342), the National Basic Research Program (973 Program) (No. 2011CB504702) and National Key Technology R\&D Program of the Ministry of Science and Technology (No. 2014BAl13B04).

The funders had no role in study design, data collection and analysis, decision to publish, or preparation of the manuscript.
Lihua Wang, Yuxi Cao, Qing Tang and Guodong Liang declare that they have no conflict of interest.

\section{REFERENCES}

Cai, L., Tao, X., Liu, Y., Zhang, H., Gao, L., et al. (2011). Biomed Environ Sci 24, $431-437$.

Centers for Disease Control Prevention. (2012). MMWR Morb Mortal Wkly Rep 61, 61-65.

Faber, M., Li, J., Kean, R.B., Hooper, D.C., Alugupalli, K.R., et al. (2009). Proc Natl Acad Sci USA 106, 11300-11305.

Fabis, M.J., Phares, T.W., Kean, R.B., Koprowski, H., Hooper, D.C. (2008). Proc Natl Acad Sci U S A 105, 15511-15516.

Galea, I., Bernardes-Silva, M., Forse, P.A., van Rooijen, N., Liblau, R.S., et al. (2007). J Exp Med 204, 2023-2030.

Hemachudha, T., Ugolini, G., Wacharapluesadee, S., Sungkarat, W., Shuangshoti, S., et al. (2013). Lancet Neurol 12, 498513.

Jackson, A.C. (2005). N Engl J Med 352, 2549-2550

Jackson, A.C. (2009). Biomedica 29, 169 176.

Kuang, Y., Lackay, S.N., Zhao, L., and Fu, Z.F. (2009). Virus Res 144, 18-26.

Laothamatas, J., Hemachuda,T., Mitrabhajdi, E., Wannakrairot, P., and Tulayadaechonont, S. (2003). AJNR Am J Neuroradiol 24, 1102-1109.

Li, J., Ertel, A., Portocarrero, C., Barkhouse, D.A., Dietzschold, B., et al. (2012). J Virol 86, 3200-3210

Liu, M. T., Keirstead, H. S., and Lane, T. E. (2001). J Immunol 167, 4091-4097.

Lv, X.J., Ma, X.J., Wang, L.H., Li, H., Shen, X.X., et al. ( 2012). Biomed Environ Sci 25, 98-103

Man, S.M., Ma, Y.R., Shang, D.S., Zhao, W.D., Li, B., et al. (2007). Neurobiol Aging 28, 485-496.

Mucke, L., and Eddleston, M. (1993). FASEB

\section{J 7,1226-1232.}

Pachter, J. S., de Vries, H. E., and Fabry, Z. (2003). J Neuropathol Exp Neurol 62, 593-604.

Phares, T.W., Fabis, M.J., Brimer, C.M., Kean, R.B., and Hooper, D.C. (2007). J Immunol 178, 7334-7343.

Phares, T.W., Kean, R.B., Mikheeva, T., and Hooper, D.C. (2006). J Immunol 176, 7666-7675.

Roy, A., and Hooper, D.C. (2007). J Virol 81, 7993-7998.

Roy, A., and Hooper, D.C. (2008). J Neurovirol 14, 401-411.

Sarmento, L., Li, X. Q., Howerth, E., Jackson, A. C., and Fu, Z. F. (2005). J Neurovirol $11,571-581$.

Stamatovic, S.M., Keep R.F., Kunkel S.L., and Andjelkovic, A.V. (2003). J Cell Sci 116, 4615-4628.

Tanabe, S., Heesen, M., Yoshizawa, I., Berman, M.A., Luo, Y. et al. (1997). J Immunol 159,905-911.

Wang, Z.W., Sarmento, L., Wang, Y., Li, X.Q., Dhingra, V., et al. (2005). J Virol 79, 12554-12565.

Wen, Y., Wang, H., Wu, H., Yang, F., Tripp, R.A., et al. (2011). J Virol 85, 1634-1644.

Willoughby, R.E., Tieves, K.S., Hoffman, G.M., and Ghanayem, N.S. (2005). N Engl J Med 352, 2508-2514.

Wu, X., Franka, R., Henderson, H., and Rupprecht, C.E. (2011). Vaccine, 29, 4195-4201.

Yin, W., Dong, J., Tu, C., Edwards, J., Guo, F., et al. (2013). Infect Dis Poverty 2, 23.

Zhao, L., Toriumi, H., Wang, H., Kuang, Y., Guo, X., et al. (2010). J Virol 84, 96429648

Zhu, W.Y., and Liang, G.D. (2012). Biomed Environ Sci 25, 602-605.

Zlotnik, A., and Yoshie, O. (2000). Immunity 12, 121-127.

Zozulya, A.L., Reinke, E., Baiu, D.C., Karman, J., Sandor, M., et al. (2007). J Immunol 178, 520-529. 